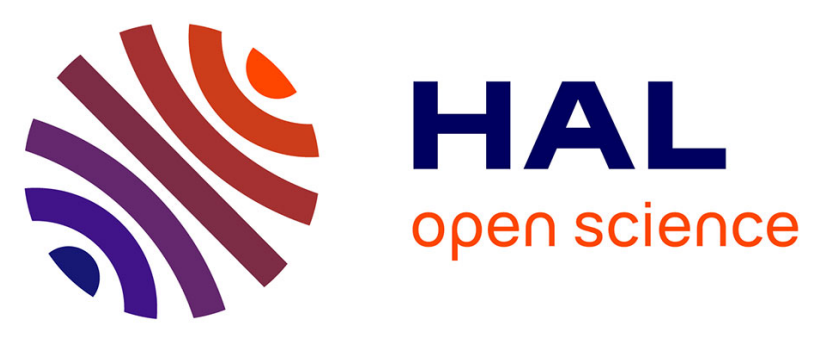

\title{
Prediction-based control for nonlinear state-and input-delay systems with the aim of delay-robustness analysis
}

Delphine Bresch-Pietri, Nicolas Petit, Miroslav Krstic

\section{- To cite this version:}

Delphine Bresch-Pietri, Nicolas Petit, Miroslav Krstic. Prediction-based control for nonlinear stateand input-delay systems with the aim of delay-robustness analysis. CDC 2015 - 54th IEEE Conference on Decision and Control, Dec 2015, Osaka, Japan. pp.6403-6409. hal-01251623

\section{HAL Id: hal-01251623 \\ https://hal.science/hal-01251623}

Submitted on 6 Jan 2016

HAL is a multi-disciplinary open access archive for the deposit and dissemination of scientific research documents, whether they are published or not. The documents may come from teaching and research institutions in France or abroad, or from public or private research centers.
L'archive ouverte pluridisciplinaire HAL, est destinée au dépôt et à la diffusion de documents scientifiques de niveau recherche, publiés ou non, émanant des établissements d'enseignement et de recherche français ou étrangers, des laboratoires publics ou privés. 


\title{
Prediction-based control for nonlinear state- and input-delay systems with the aim of delay-robustness analysis
}

\author{
Delphine Bresch-Pietri, Nicolas Petit and Miroslav Krstic
}

\begin{abstract}
This paper investigates prediction-based control for nonlinear systems subject to both pointwise input- and (potentially) distributed state-delays. We address infinity-norm stability analysis of the corresponding closed-loop system reformulating both delays as transport Partial Differential Equations (PDEs) and transforming the resulting distributed state. We show how the performed analysis can be extended to establish robustness to delay uncertainties. We illustrate the merit of this design with numerical simulation of a prey-predator population dynamics.
\end{abstract}

\section{INTRODUCTION}

Time-delays systems are often involved in the modeling of systems exhibiting periodical behaviors, as it is the case in biology [25] or population dynamics [4], [11], [14] for example. This lag, which could be either pointwise or distributed, accounts for physical distance and related transport, blood circulation, cell generation, etc. Most of the time, those delays affect both the input and the state. The same conclusion applies, e.g., for systems involving a recycle loop as it is commonly the case in the process industry (see [22], [10], [8]). While most of the recycle processes can reasonably be modeled as linear dynamics, this is not the case of most biological systems.

In this paper, we design a prediction-based controller for nonlinear systems subject to both input and state delays. Predictor-based control strategies, more commonly known as Smith Predictor (see [1],[28],[21]) are state-ofthe-art for systems with constant input time-delays (see for instance [3],[5],[12],[13],[23], or [27] and the reference therein). Grounding on the use of a prediction of the system state on a time horizon equal to the input delay, this technique aims at compensating this delay, which notably improves the transient performances. However, while the literature on control of either input delay or state delay systems is extremely wealth, systems with both input and state delays have seldom been studied, except from very recent contributions [2], [9], [17].

In this work, we consider nonlinear dynamics with (potentially) distributed state delay subject to pointwise inputdelay. We assume that we know a nominal feedback law which stabilizes the input-delay-free nominal system (and potentially compensates the state delay by relying on past

D. Bresch-Pietri (corresponding author) is with CNRS at the Department of Automatic Control, Gipsa-lab, 11 rue des Mathématiques, 38000 Grenoble, France Email: delphine.bresch-pietri@gipsa-lab.fr

N. Petit is with MINES ParisTech, PSL Research University, CAS Centre automatique et systèmes, 60 bd St Michel 75006 Paris, France

M. Krstic is with the Department of Mechanical and Aerospace Engineering, University of California, San Diego, La Jolla CA 92093, USA values of the system state). The strategy that we advocate is similar to the one proposed in [2]. It consists in the use of such a nominal feedback law, which, instead of being applied to the current state, takes as input a suitable prediction of the system state.

While the control design and the stability result we propose are very close to the ones in [2], the stability analysis we present relies on a different methodology which enables one to pursue a robustness analysis in the future. In details, our approach is grounded on PDEs tools that were proposed lately to address input delay uncertainties (see [19], [20]) and were extended in [9] to handle both state- and inputdelay uncertainties. In this paper, we build on those previous contributions to propose a PDE framework accounting for nonlinearities and distributed delays. Modeling both actuator and the state delays as transport PDEs coupled with the original Ordinary Differential Equation (ODE), we rely on a backstepping transformation of the distributed input to analyze the closed-loop stability. To formulate the corresponding target system, one needs to study an implicit functional PDE. While nonlinearity is responsible for the implicit nature, the functional one originates from the fact that the state-delay is distributed. We then carry out an $\mathscr{L}_{\infty}$ analysis for the closedloop system. This is the main contribution of the paper.

While we do not formally investigate the delay-robustness problem, we show how the proposed methodology can be applied to analyze it. The objective of this paper is to present this new PDE framework by providing an alternative proof to the one in [2] as a preliminary step.

The paper is organized as follows. In Section II, we introduce the problem under consideration before providing the prediction-based control we propose in Section III. Then, we present the stability analysis of the closed-loop dynamics in Section IV before sketching in Section V how it can be extended to analyze robustness to delay uncertainties. Finally, we illustrate the merits of our designs in Section VI with simulations of a prey-predator model.

Notations. Let $n$ be a positive integer and $\mu$ a given positive constant. In the sequel, for $u \in \mathscr{C}\left([0,1] \times \mathbb{R}, \mathbb{R}^{n}\right)$ and $\varphi \in \mathscr{C}\left(I, \mathbb{R}^{n}\right)$ for any interval $I \subset \mathbb{R}$, we denote

$$
\begin{aligned}
\|u(t)\|_{p, \mu} & =\left(\int_{0}^{1} e^{p \mu x}|u(x, t)|^{p} d x\right)^{\frac{1}{p}} \\
\|u(t)\|_{\infty, \mu} & =\max _{x \in[0,1]}\left|e^{\mu x} u(x, t)\right|=\lim _{p \rightarrow \infty}\|u(t)\|_{p, \mu} \\
\|\varphi\|_{\infty} & =\max _{s \in I}|\varphi(s)|
\end{aligned}
$$

and we use the standard notations $\|\cdot\|_{p}=\|\cdot\|_{p, 0}$ 
and $\quad\|\cdot\|_{\infty}=\|\cdot\|_{\infty, 0}$. For $\left(D_{1}, D_{2}\right) \in \mathbb{R}^{2} \quad$ and a function $X$, we write $X_{t, 1}: s \in\left[-D_{1}, 0\right] \mapsto X(t+s)$ and $\quad X_{t, 2}: s \in\left[-D_{2}, 0\right] \mapsto X(t+s)$. Finally, for $u \in \mathscr{C}\left([0,1] \times \mathbb{R}, \mathbb{R}^{n}\right)$, we define $u(\cdot, t): x \in[0,1] \mapsto u(x, t)$ for $t \geq 0$ and, for $v \in \mathscr{C}\left([0,1]^{2} \times \mathbb{R}, \mathbb{R}^{n}\right)$, we define $v(x, \cdot, t): y \in[0,1] \mapsto v(x, y, t)$ for $(x, t) \in[0,1] \times \mathbb{R}$ and $v(\cdot, \diamond, t):(x, y) \in[0,1]^{2} \mapsto v(x, y, t)$ for $(x, t) \in[0,1] \times \mathbb{R}$.

\section{Problem Statement}

Consider the (potentially) unstable system

$$
\dot{X}(t)=f\left(X_{t, 1}, U\left(t-D_{2}\right)\right)
$$

in which $X \in \mathbb{R}^{n}, U$ is scalar and $D_{2}>D_{1}>0$. In the following, we assume that $f$ is locally Lipschitz and that its partial derivative with respect to its first argument (Gâteaux derivative) is locally Lipschitz.

The dynamics (4) can encompass various complex delay classes. Those can be either distributed or pointwise (however, we do not allow the delay to be time-varying ${ }^{1}$ ).

The control objective is to stabilize (4) following a prediction-based approach to compensate the input delay. With this aim in view, we first characterize further the system under consideration.

Assumption 1: The system (4) is forward complete.

Assumption 2: There exists a feedback law $\kappa: \mathscr{C}^{0}\left(\left[-D_{1}, 0\right], \mathbb{R}^{n}\right) \mapsto \mathbb{R}$ which is a class $\mathscr{C}^{1}$ function such that the dynamics

$$
\dot{X}(t)=f\left(X_{t, 1}, \kappa\left(X_{t, 1}\right)\right)
$$

is globally exponentially stable, i.e.(see [18], [26]), there exist a continuous functional $W: \mathscr{C}\left(\left[-D_{1}, 0\right], \mathbb{R}^{n}\right)$ and constants $C_{1}, C_{2}, C_{3}>0$ such that

$$
\begin{aligned}
& C_{1}\|\varphi\|_{\infty} \leq W(\varphi) \leq C_{2}\|\varphi\|_{\infty} \\
& \left|\partial_{\varphi} W(\varphi)\right| \leq C_{3}
\end{aligned}
$$

and, moreover, the functional $W$ is differentiable along the trajectories of the closed-loop system (5) and

$$
\dot{W}(t) \leq-W(t)
$$

Assumption 1 guarantees that (4) does not escape in finite time and, in particular, before the closed-loop input reaches the system at time $t=D_{2}$. This is a necessary assumption to guarantee that the control designed in the sequel will stabilize the plant for any delay value.

Assumption 2 guarantees the existence of a nominal feedback law for the input-delay free system. We assume that this feedback law is exponentially stabilizable. Alternatively, one could consider an asymptotically stabilizable feedback law as it is done in [2]. However, the nominal closed-loop dynamics should then also satisfy an Input-to-State Stability property, which is not needed here. Note that we consider

\footnotetext{
${ }^{1}$ Even if the methodology we propose could be extended to statedependent delays or to time-varying delays a model of which is known. This is a direction of future work.
}

that this feedback map is a function of the distributed state $X_{t, 1}$ to allow state-delay compensation.

In the sequel, we build on this nominal feedback law and design a prediction strategy to compensate the input-delay.

\section{NOMINAL CONTROL DESIGN}

Consider the distributed state prediction

$$
\begin{aligned}
& P^{t}(\tau)= \\
& \begin{cases}X\left(\tau+D_{2}\right) & \text { if } t-D_{1}-D_{2} \leq \tau \leq t-D_{2} \\
X(t)+\int_{t-D_{2}}^{\tau} f\left(P_{s, 1}^{t}, U(s)\right) d s & \text { if } t-D_{2} \leq \tau \leq t\end{cases}
\end{aligned}
$$

for $t \geq 0$ and $\tau \in\left[t-D_{1}-D_{2}, t\right]$. We now use this prediction as argument for the nominal input-delay free control law in lieu of the original distributed state

$$
U(t)=\kappa\left(P_{t, 1}^{t}\right)
$$

Theorem 1: Consider the closed-loop system consisting of the plant (4) satisfying Assumptions 1-2 and the control law (10) involving the prediction (9). Define the functional

$$
\Gamma(t)=\left\|X_{t, 1}\right\|_{\infty}+\left\|U_{t, 2}\right\|_{\infty}
$$

There exists a class $\mathscr{K} \mathscr{L}$ function $\beta$ such that, for $\left(X_{0,1}, U_{0,2}\right) \in \mathscr{C}^{0}\left(\left[-D_{2}, 0\right]\right) \times \mathscr{C}^{0}\left(\left[-D_{1}, 0\right]\right)$,

$$
\Gamma(t) \leq \beta(\Gamma(0), t), t \geq 0
$$

In order to properly understand the choice of the control law, we provide several comments next.

First, it is worth noticing that $P_{t, 1}^{t}$ defined through (9) is a $D_{2}$ units of time ahead prediction of the distributed system state $X_{t, 1}$. Indeed, integrating (4) between $t$ and $\tau+D_{2}$, one obtains

$$
\begin{aligned}
X\left(\tau+D_{2}\right) & =X(t)+\int_{t}^{\tau+D_{2}} f\left(X_{s, 1}, U\left(s-D_{2}\right)\right) d s \\
& =X(t)+\int_{t-D_{2}}^{\tau} f\left(X_{s+D_{2}-D_{1}, 1}, U(s)\right) d s
\end{aligned}
$$

in which the second equation has been obtained performing a change of variable. One can observe that this equation is equivalent to (9). Hence, formally, one obtains $P^{t}(\tau)=$ $X\left(\tau+D_{2}\right)$ for $\tau \in\left[t-D_{1}, t\right]$ as $D_{1}<D_{2}$. Plugging this control law into the original dynamics, one naturally infers that the closed-loop dynamics should be (5) which is asymptotically stable by assumption.

Second, note that we define the prediction as a function of two arguments: $P^{t_{1}}(\tau)$ is the prediction $X\left(\tau+D_{2}\right)$ computed at time $t_{1}$ and using $X\left(t_{1}\right)$ as a starting point. A priori, this prediction could be different from $P^{t_{2}}(\tau)$ for a different $t_{2}$. However, this is not the case here, as we consider no model uncertainty, but it is likely to be the case in practical applications. In particular, for the nominal case under consideration here, the definition of the distributed prediction (9) is formally equivalent to the following one 
given in [2]

$$
\begin{aligned}
& P(\tau)=X(t)+\int_{t-D_{2}}^{\tau} f\left(P_{s, 1}, U(s)\right) d s, \\
& \text { for } t-D_{2} \leq \tau \leq t \text { with } t>D_{2} \\
& P(\tau)= \\
& \left\{\begin{array}{lr}
X\left(\tau+D_{2}\right) & \text { if }-D_{1}-D_{2} \leq \tau \leq-D_{2} \\
X(0)+\int_{-D_{2}}^{\tau} f\left(P_{s, 1}, U(s)\right) d s & \text { if }-D_{2} \leq \tau \leq 0
\end{array}\right.
\end{aligned}
$$

in which $P$ is then expressed as a function of one argument to emphasize the fact that it actually does not depend on the time at which the computation is done. However, (9) constantly incorporates measured delayed state while (14)(15) is initialized with them and then only integrates the dynamics. Thus, in all likelihood, the formulation we propose should improve the robustness of the prediction to model mismatch or delay uncertainty (even if the induced number of computations would then increase). Further, it facilitates the stability analysis, as will appear in the following.

Finally, even if the equation (9) may seem implicit at first glance, this prediction is actually well-defined and the solution always exists and is unique, as the solution of the differential equation (4). Further, more interestingly, it is also practically computable, relying on suitable discretization scheme of the integral (see [29] for a study on the effect of this discretization scheme on the closed-loop stability of linear systems and [16] where nonlinear dynamics are addressed and a time-varying discretization methodology is proposed. Alternatively, one can rely on a a low-pass filter addition [24] for linear systems or on an approximate predictor as done in [15].).

We now provide an alternative proof to this theorem, relying on PDEs reformulation.

\section{Proof of Theorem 1 With PDEs technique}

\section{A. PDEs reformulation and backstepping transformation}

As a first step in our analysis, we define the distributed variables $\zeta(x, t)=X\left(t+D_{1}(x-1)\right)$ and $u(x, t)=U\left(t+D_{2}(x-1)\right)$ for $x \in[0,1]$. The plant (4) can then be reformulated as the following ODE-PDE cascade

$$
\left\{\begin{aligned}
\dot{X}(t) & =f_{0}(\zeta(\cdot, t), u(0, t)) \\
D_{1} \partial_{t} \zeta(x, t) & =\partial_{x} \zeta(x, t) \\
\zeta(1, t) & =X(t) \\
D_{2} \partial_{t} u(x, t) & =\partial_{x} u(x, t) \\
u(1, t) & =U(t)
\end{aligned}\right.
$$

in which we used

$$
f_{0}(\zeta(\cdot, t), u(0, t))=f\left(X_{t, 1}, u(0, t)\right)
$$

which is a simple change of arguments in the function definition. Similarly, in the sequel, we denote

$$
\kappa_{0}(\zeta(\cdot, t))=\kappa\left(X_{t, 1}\right)
$$

Now define a distributed prediction, for $x \in[0,1]$ and $t \geq 0$,

$$
\begin{gathered}
p(x, t)=X(t)+D_{2} \int_{0}^{x} f_{0}(\chi(y, \cdot, t), u(y, t)) d y \\
\chi(x, y, t)= \begin{cases}\zeta\left(y+x \frac{D_{2}}{D_{1}}, t\right) & \text { if } x D_{2}+(y-1) D_{1} \leq 0 \\
p\left(x+\frac{D_{1}}{D_{2}}(y-1), t\right) & \text { if } x D_{2}+(y-1) D_{1} \geq 0\end{cases} \\
\text { for } 0 \leq y \leq 1
\end{gathered}
$$

along with the following backstepping transformation of the distributed input $u(x, t)$

$$
w(x, t)=u(x, t)-\kappa_{0}(\chi(x, \cdot, t)), 0 \leq x \leq 1
$$

Lemma 1: The infinite-dimensional backstepping transformation (21) together with the control law (10) transform (16) into the target system

$$
\begin{aligned}
\dot{X}(t) & =f_{0}\left(\zeta(\cdot, t), \kappa_{0}(\zeta(\cdot, t))+w(0, t)\right) \\
D_{1} \partial_{t} \zeta(x, t) & =\partial_{x} \zeta(x, t) \\
\zeta(1, t) & =X(t) \\
D_{2} \partial_{t} w(x, t) & =\partial_{x} w(x, t) \\
w(1, t) & =0
\end{aligned}
$$

Proof: We start this proof by noticing that $p(x, t)=P^{t}\left(t+D_{2}(x-1)\right)$ for $x \in[0,1]$ and that $\chi(x, y, t)=P^{t}\left(t+D_{2}(x-1)+D_{1}(y-1)\right)$ for $(x, y) \in[0,1]^{2}$ (this could be obtained performing a change of variable in (19) and using the fact that the solution to the differential equation (4) or, equivalently, to the integral equation (9) is unique). From there, it follows that $U(t)=\kappa_{0}(\chi(1, \cdot, t))$. Consequently, from the definition (21), one obtains (26). Further, from (20), one gets

$$
\chi(0, y, t)=\zeta(y, t)
$$

and (22) follows with (21).

Now, taking space- and time-derivatives of (19), one obtains

$$
\begin{aligned}
\partial_{t} p(x, t)= & f\left(X_{t, 1}, u(0, t)\right) \\
& +D_{2} \int_{0}^{x}\left(\frac{\partial f_{0}}{\partial \chi}(\chi(y, \cdot, t), u(y, t)) \cdot \partial_{t} \chi(y, \cdot, t)\right. \\
& \left.+\frac{\partial f_{0}}{\partial u}(\chi(y, \cdot, t), u(y, t)) \cdot \partial_{t} u(y, t)\right) d y \\
\partial_{x} p(x, t)= & D_{2} f_{0}(\chi(x, \cdot, t), u(x, t)) \\
= & D_{2}\left(f_{0}(\chi(0, \cdot, t), u(0, t))\right. \\
& +\int_{0}^{x}\left(\frac{\partial f}{\partial \chi}(\chi(y, \cdot, t), u(y, t)) \cdot \partial_{x} \chi(y, \cdot, t)\right. \\
& \left.\left.+\frac{\partial f}{\partial u}(\chi(y, \cdot, t), u(y, t)) \cdot \partial_{x} u(y, t)\right) d y\right)
\end{aligned}
$$

Observing that $\chi(0, \cdot, t)=\zeta(\cdot, t)$ from (20) and that $f_{0}(\zeta(\cdot, t), u(0, t))=f\left(X_{t, 1}, u(0, t)\right)$, it follows that

$$
\begin{aligned}
& D_{2} \partial_{t} p(x, t)-\partial_{x} p(x, t)= \\
& D_{2} \int_{0}^{x} \frac{\partial f_{0}}{\partial \chi}(\chi(y, \cdot, t), u(y, t)) \cdot\left(D_{2} \partial_{t} \chi(y, \cdot, t)-\partial_{x} \chi(y, \cdot, t)\right) d y
\end{aligned}
$$


Now, taking spatial- and time-derivatives of (20), one gets

$$
\begin{aligned}
& \partial_{t} \chi(x, y, t)= \\
& \begin{cases}\partial_{t} \zeta\left(y+x \frac{D_{2}}{D_{1}}, t\right) & \text { if } x D_{2}+(y-1) D_{1}<0 \\
\partial_{t} p\left(x+\frac{D_{1}}{D_{2}}(y-1), t\right) & \text { if } x D_{2}+(y-1) D_{1}>0\end{cases} \\
& \partial_{x} \chi(x, y, t)= \\
& \begin{cases}\frac{D_{2}}{D_{1}} \partial_{x} \zeta\left(y+x \frac{D_{2}}{D_{1}}, t\right) & \text { if } x D_{2}+(y-1) D_{1}<0 \\
\partial_{x} p\left(x+\frac{D_{1}}{D_{2}}(y-1), t\right) & \text { if } x D_{2}+(y-1) D_{1}>0\end{cases}
\end{aligned}
$$

and, thus, using (23) and (30),

$$
\begin{aligned}
& D_{2} \partial_{t} \chi(x, y, t)-\partial_{x} \chi(x, y, t)= \\
& \left\{\begin{array}{l}
0 \\
D_{2} \int_{0}^{x+\frac{D_{1}}{D_{2}}(y-1)} \frac{\partial f_{0}}{\partial \chi}(\chi(\xi, \cdot, t), u(\xi, t)) \\
\cdot\left(D_{2} \partial_{t} \chi(\xi, \cdot, t)-\partial_{x} \chi(\xi, \cdot, t)\right) d \xi \text { if } x D_{2}+(y-1) D_{1}>0
\end{array}\right.
\end{aligned}
$$

Thus, defining $r(x, \cdot, t)=D \partial_{t} \chi(x, \cdot, t)-\partial_{x} \chi(x, \cdot, t)$ and, for $(s, x, t) \in\left[-D_{1} / D_{2}, 0\right] \times[0,1] \times \mathbb{R}$,

$$
r_{x}(s, \cdot, t)= \begin{cases}r(s+x, \cdot, t) & \text { if } s+x \geq 0 \\ 0 & \text { otherwise }\end{cases}
$$

there exists a continuous function $g:[0,1] \times \mathbb{R} \rightarrow$ $\mathscr{C}\left(\mathscr{C}\left(\left[-D_{1} / D_{2}, 0\right] \times[0,1] \times \mathbb{R}, \mathbb{R}\right), \mathscr{C}([0,1] \times \mathbb{R}, \mathbb{R})\right) \quad$ such that

$$
\begin{aligned}
\partial_{x} r(x, \cdot, t) & =g(x, t) \cdot r_{x}(\cdot, \diamond, t) \\
r_{0}(\cdot, \diamond, t) & =0
\end{aligned}
$$

As the plant (4) is forward complete, $p$ and thus $\chi$ exist for all time. Hence, the functional $g$ is defined for all time. From there, one obtains that the solution of this system is global and unique. Solving this equation, it follows that $r(x, t)=0$ or, equivalently,

$$
D_{2} \partial_{t} \chi(x, \cdot, t)=\partial_{x} \chi(x, \cdot, t)
$$

Finally, taking space- and time-derivatives of (21), one obtains

$$
\begin{aligned}
& \partial_{t} w(x, t)=\partial_{t} u(x, t)-\frac{d \kappa}{d \chi}(\chi(x, \cdot, t)) \cdot \partial_{t} \chi(x, \cdot, t) \\
& \partial_{x} w(x, t)=\partial_{x} u(x, t)-\frac{d \kappa}{d \chi}(\chi(x, \cdot, t)) \cdot \partial_{x} \chi(x, \cdot, t)
\end{aligned}
$$

Therefore, with (16) and (37), the transport PDE (25) follows straightforwardly.

We now exploit the backstepping transformation (21) to provide a Lyapunov analysis of the stability of the closedloop system. The reason for this choice is linked to the boundary condition (26) as will appear in the sequel.

\section{B. Lyapunov analysis}

Define the following Lyapunov-Krasovskii functional candidate

$$
\begin{aligned}
V_{p}(t) & =\left(\mu-1+\frac{1}{2 p}\right) W(t)^{2 p} \\
& +D b_{p}^{2 p}\left(\left\|X_{0,1}\right\|_{\mu, 2 p}+\|w(0)\|_{\mu, 2 p}\right) \int_{0}^{1} e^{2 p \mu x} w(x, t)^{2 p} d x
\end{aligned}
$$

in which the functional $W$ has been introduced in Assumption $2, p \in \mathbb{N}, \mu>1$ and

$$
\begin{aligned}
& b_{p}\left(\left\|X_{0,1}\right\|_{\mu, 2 p}+\|w(0)\|_{\mu, 2 p}\right)= \\
& \quad \max \left\{\left(\mu-1+\frac{1}{2 p}\right) C_{3} \ell\left(\left\|X_{0,1}\right\|_{\mu, 2 p}+\|w(0)\|_{\mu, 2 p}\right), 1\right\}
\end{aligned}
$$

and $\ell: \mathbb{R}_{+} \rightarrow \mathbb{R}_{+}$is defined as the Lipschitz constant of $f(\cdot, \kappa(\cdot))$ with respect to its second argument over the ball $B(0, R)$. In the following, for the sake of clarity, we omit the arguments of $b_{p}$ and $\ell$.

Taking a time-derivative of this functional and using integrations by parts, one obtains

$$
\begin{aligned}
\dot{V}_{p}(t)= & (2 p(\mu-1)+1) W^{2 p-1} \\
& \times \partial_{X_{t, 1}} W\left(X_{t, 1}\right) \cdot f\left(X_{t, 1}, \kappa\left(X_{t, 1}\right)+w(0, t)\right) \\
& -2 p \mu b_{p}^{2 p} \int_{0}^{1} e^{2 p \mu x} w(x, t)^{2 p} d x-b_{p}^{2 p} w(0, t)^{2 p} \\
\leq & -(2 p(\mu-1)+1) W(t)^{2 p}-2 p \mu b_{p}^{2 p}\|w(t)\|_{2 p, \mu}^{2 p} \\
& +(2 p(\mu-1)+1) W^{2 p-1} \ell C_{3}|w(0, t)|-b_{p}^{2 p} w(0, t)^{2 p}
\end{aligned}
$$

in which we have also used (7) and (8). Further, using Young inequality, one obtains

$$
\begin{aligned}
& (2 p(\mu-1)+1) W^{2 p-1} \ell C_{3}|w(0, t)| \\
& \quad \leq(2 p-1) W(t)^{2 p}+\left(\mu-1+\frac{1}{2 p}\right)^{2 p} C_{3}^{2 p} \ell^{2 p} w(0, t)^{2 p}
\end{aligned}
$$

Therefore, plugging (43) into (42) and using (41), it follows

$$
\dot{V}_{p}(t) \leq-2 p \mu V_{p}(t), t \geq 0
$$

and thus, for $t \geq 0$,

$$
\begin{aligned}
V_{p}(t)^{\frac{1}{2 p}} \leq e^{-\mu t} & \left(\left(\left(\mu-1+\frac{1}{2 p}\right)^{\frac{1}{2 p}} W(0)\right.\right. \\
& \left.+b_{p}\left(D \int_{0}^{1} e^{2 p \mu x} w(x, 0)^{2 p} d x\right)^{\frac{1}{2 p}}\right)
\end{aligned}
$$

This gives, for $t \geq 0$,

$$
\begin{aligned}
& (\mu-1)^{\frac{1}{2 p}} W(t)+b_{p}\left(D \int_{0}^{1} e^{2 p \mu x} w(x, t)^{2 p} d x\right)^{\frac{1}{2 p}} \\
& \leq 2 e^{-\mu t}\left(\left(\mu-\frac{1}{2}\right){ }^{\frac{1}{2 p}}(0)+b_{p}\left(D \int_{0}^{1} e^{2 p \mu x} w(x, 0)^{2 p} d x\right)^{\frac{1}{2 p}}\right)
\end{aligned}
$$

Now, define

$$
\begin{aligned}
\Gamma_{0}(t) & =W(t)+b\|w(t)\|_{\mu, \infty} \\
\tilde{\Gamma}(t) & =W(t)+\|w(t)\|_{\infty}
\end{aligned}
$$

in which $b \triangleq \max \left\{(\mu-1) C_{3} \ell\left(\left\|X_{0,1}\right\|_{\infty}+\|w(0)\|_{\infty}\right), 1\right\}$. Taking the limit of (46) as $p$ tends to infinity, one finally gets

$$
\Gamma_{0}(t) \leq 2 e^{-\mu t} \Gamma_{0}(t), t \geq 0
$$

and thus, using (6) and the fact that $b \geq 1$ by definition, the existence of a $\mathscr{K}_{\infty}$ function $\tilde{\alpha}$ such that

$$
\tilde{\Gamma}(t) \leq \tilde{\alpha}(\tilde{\Gamma}(0)) e^{-\mu t}
$$




\section{Equivalence between functionals $\Gamma$ and $\tilde{\Gamma}$}

As a final step to prove Theorem 1, the fact that $\Gamma$ in (11) and $\tilde{\Gamma}$ in (48) are equivalent remains to be shown. With this aim in view, we first prove the following lemma.

Lemma 2: There exist class $\mathscr{K}_{\infty}$ functions $\alpha_{1}$ and $\alpha_{2}$ such that

$$
\begin{aligned}
\|u(t)\|_{\infty} & \leq \alpha_{1}\left(\left\|X_{t, 1}\right\|_{\infty}+\|w(t)\|_{\infty}\right) \\
\|w(t)\|_{\infty} & \leq \alpha_{2}\left(\left\|X_{t, 1}\right\|_{\infty}+\|u(t)\|_{\infty}\right)
\end{aligned}
$$

Proof: From (19) and (20), there exists a class $\mathscr{K}_{\infty}$ function $\alpha_{3}$ such that

$$
\|\chi(t)\|_{\infty} \leq \alpha_{3}\left(\left\|X_{t, 1}\right\|_{\infty}+\|u(t)\|_{\infty}\right)
$$

Second, from (21), one obtains the existence of a class $\mathscr{K}_{\infty}$ function $\alpha_{4}$ such that

$$
\|w(t)\|_{\infty} \leq \alpha_{4}\left(\|u(t)\|_{\infty}+\|\chi(t)\|_{\infty}\right)
$$

Gathering (53)-(54), one obtains the existence of $\alpha_{2}$ such that (52) holds. The one of $\alpha_{1}$ such that (51) holds follows similar lines.

Now, observing that,

$$
\begin{aligned}
\|u(t)\|_{\infty} & =\max _{x \in[0,1]}\left|U\left(t+D_{2}(x-1)\right)\right| \\
& =\max _{s \in\left[t-D_{2}, t\right]}|U(s)|=\left\|U_{t, 2}\right\|_{\infty}
\end{aligned}
$$

one gets, using (6) and Lemma 2, the existence of class $\mathscr{K}_{\infty}$ functions $\alpha_{5}$ and $\alpha_{6}$ such that

$$
\alpha_{5}(\tilde{\Gamma}(t)) \leq \Gamma(t) \leq \alpha_{6}(\tilde{\Gamma}(t))
$$

From there, using (50), one obtains the existence of a class $\mathscr{K} \mathscr{L}$ function $\beta$ such that Theorem 1 holds. This concludes the proof.

\section{ON DELAY-ROBUSTNESS OF THE PREDICTION-BASED CONTROL LAW (9)-(10)}

In this section, we investigate how the previously proposed design can be extended in the case of (state- and input-) delays uncertainty. We start by formulating a new assumption.

Assumption 3: There exist scalars $\underline{D}$ and $\bar{D}$ such that $0<$ $\underline{D}<D_{1}<D_{2}<\bar{D}$.

We now consider that the two delays are uncertain and use $\hat{D}_{1}$ and $\hat{D}_{2}$ as state-delay and input-delay estimates, respectively (with $\left.\left(\hat{D}_{1}, \hat{D}_{2}\right) \in[\underline{D}, \bar{D}]^{2}\right)$. The previously proposed design is then modified as follows.

\section{A. Control design}

The estimated distributed state prediction is now

$$
\begin{aligned}
& \hat{P}^{t}(\tau)= \\
& \left\{\begin{array}{l}
X\left(\tau+\hat{D}_{2}\right) \\
X(t)+\int_{t-\hat{D}_{2}}^{\tau} f\left(\hat{P}_{s, 1}^{t}, U(s)\right) d s \quad \text { if } t-\hat{D}_{1}-\hat{D}_{2} \leq \tau \leq t-\hat{D}_{2}
\end{array}\right.
\end{aligned}
$$

for $t \geq 0$ and $\tau \in\left[t-\hat{D}_{1}-\hat{D}_{2}, t\right]$ and the control law is

$$
U(t)=\kappa\left(\hat{P}_{t, 1}^{t}\right)
$$

Claim 1: Consider the closed-loop system consisting of the plant (4) satisfying Assumptions 1-2 and the control law (10) involving the prediction (9). Define the functional

$$
\begin{aligned}
& \Gamma(t)=\max _{s \in\left[-\max \left\{D_{1}, 2 \hat{D}_{1}\right\}, 0\right]}|X(t+s)|+\max _{s \in\left[-\hat{D}_{2}, 0\right]}|\ddot{U}(t)| \\
& +\max _{s \in\left[-\max \left\{D_{2}, \hat{D}_{1}+\hat{D}_{2}\right\}, 0\right]}|U(t+s)|+\max _{s \in\left[-\max \left\{D_{2}, \hat{D}_{2}\right\}, 0\right]}|\dot{U}(t+s)|
\end{aligned}
$$

Consider $\left.\left(X_{0,1}, U_{0,2}\right) \in \mathscr{C}^{1}\left[-D_{2}, 0\right]\right) \times \mathscr{C}^{2}\left(\left[-D_{1}, 0\right]\right)$. There exist positive functions $\Delta_{1}^{*}(\Gamma(0))$ and $\Delta_{2}^{*}(\Gamma(0))$ such that, if $\left|D_{1}-\hat{D}_{1}\right|<\Delta_{1}^{*}(\Gamma(0))$ and if $\left|D_{2}-\hat{D}_{2}\right|<\Delta_{2}^{*}(\Gamma(0))$, there exists a class $\mathscr{K} \mathscr{L}$ function $\beta$ such that

$$
\Gamma(t) \leq \beta(\Gamma(0), t), t \geq 0
$$

Further, the functions $\Delta_{1}^{*}$ and $\Delta_{2}^{*}$ are decreasing functions.

\section{B. Elements of proof of Claim 1 and discussion}

For the sake of clarity, we do not provide here the proof of Claim 1 which is lengthy and technical. However, we aim at underlying its milestones which should lead to the above statement.

Grounding on [9], we propose to rely on the estimated distributed input $\hat{u}(x, t)=U\left(t+\hat{D}_{2}(x-1)\right)$ along with the estimated distributed state $\hat{\zeta}=X\left(t+\hat{D}_{1}(x-1)\right)$ to carry out the analysis. Then, one can introduce as in the previous section the backstepping transformation

$$
\hat{w}(x, t)=\hat{u}(x, t)-\kappa_{0}(\hat{\chi}(x, \cdot, t))
$$

in which the distributed prediction is now

$$
\begin{gathered}
\hat{p}(x, t)=X(t)+\hat{D}_{2} \int_{0}^{x} f_{0}(\hat{\chi}(y, \cdot, t), \hat{u}(y, t)) d y \\
\hat{\chi}(x, y, t)= \begin{cases}\hat{\zeta}\left(y+x \frac{\hat{D}_{2}}{\hat{D}_{1}}, t\right) & \text { if } x \hat{D}_{2}+(y-1) \hat{D}_{1} \leq 0 \\
\hat{p}\left(x+\frac{\hat{D}_{1}}{\hat{D}_{2}}(y-1), t\right) & \text { if } x \hat{D}_{2}+(y-1) \hat{D}_{1} \geq 0 \\
\text { for } 0 \leq y \leq 1\end{cases}
\end{gathered}
$$

This would lead to a target system similar to (22)-(26) but including transport equations corresponding to distributed errors (distributed state and input errors) and with additive source terms. To handle those source terms and the nonlinearity of the systems, it is necessary to carry out the analysis with a modified $\mathscr{H}_{2}^{p}$-norm as done in [7] (for $p=1$ ) instead of a modified $\mathscr{L}^{p}$ norm as it is the case in the previous section. Hence, the conclusion obtained in [7] for inputdelay systems would extend to the state- and input-delays case under the form of Claim 1.

In a nutshell, elements and techniques from [7], [9] and the infinity norm analysis carried out in the previous section yield the proof of Claim 1. 


\section{APPLICATION TO POPUlATION DYNAMiCS - NUMERICAL SIMULATIONS}

Time-delays are of particular interest for logistic and populations modeling as they are capable of generating periodic solutions of considerable complexity, dynamics which are often observed in those fields.

To illustrate the interest of the proposed approach, we consider the following (simplified) prey-predator model with predator harvesting

$$
\left\{\begin{array}{l}
\dot{X}_{1}=X_{1}(t)\left[g\left(X_{1}(t)\right)-X_{2}\left(t-D_{1}\right) p\left(X_{1}(t)\right)\right] \\
\dot{X}_{2}=-d X_{2}(t)+X_{1}(t) X_{2}\left(t-D_{1}\right) p\left(X_{1}(t)\right)+U\left(t-D_{2}\right)
\end{array}\right.
$$

in which $X_{1}$ is the population of preys, $X_{2}$ the predators one, $g$ the growth rate of the preys in the absence of predators, $x p(x)$ is the response function and $d$ is the death rate of predators in the absence of prey. In the above, the state delay $D_{1}$ accounts for the fact that the predation requires maturity of individuals (young predators do not kill preys); the delay $D_{2}$ accounts for the fact that the predators are neither introduced at the same physical location nor harvested before a certain size/age. Following [14], we consider $g(x)=1-\frac{x}{50}$ and $p(x)=\frac{2}{10+x}$.

The dynamics (64) satisfy Assumption 1 and exhibit a nonzero equilibrium point $X^{r}$ with $X_{1}^{r}$ solution of the equation $X_{1}^{r} p\left(X_{1}^{r}\right)=d$ and $X_{2}^{r}=g\left(X_{1}^{r}\right) / p\left(X_{1}^{r}\right)$. Furthermore, using the backstepping technique for ODEs, one obtains that a feedback law satisfying Assumption 2 is

$$
\begin{gathered}
\kappa\left(X_{t, 1}\right)=d X_{2}(t)-X_{1}(t) X_{2}\left(t-D_{1}\right) p\left(X_{1}(t)\right) \\
-\lambda_{2}\left(X_{2}(t)-v(t)\right)+\dot{v}(t) \\
v(t)=\frac{1}{\Pi(t) p_{1}(\Pi(t))}\left(\Pi(t) g(\Pi(t))+\lambda_{1}\left(\Pi(t)-X_{1}^{r}\right)\right)
\end{gathered}
$$

in which $\lambda_{1}$ and $\lambda_{2}$ are given positive constants and

$$
\Pi(\tau)=X_{1}+\int_{t-D_{1}}^{\tau} \Pi(s)\left(g(\Pi(s))-X_{2}(s) p(\Pi(s))\right) d s
$$

for $\tau \in\left[t-D_{1}, t\right]$ which only depends on $X_{t, 1}$. The timederivative $\dot{v}$ in (65) can be formally calculated using (67) and the first component of (64).

In details, this feedback law aims at compensating the state-delay through the prediction $\Pi=X_{1}\left(t+D_{1}\right)$ and the corresponding nominal input-delay-free closed-loop system is then

$\dot{X}_{1}=-\lambda_{1}\left(X_{1}(t)-X_{1}^{r}\right)-X_{1}(t) p\left(X_{1}(t)\right)\left(X_{2}\left(t-D_{1}\right)-v\left(t-D_{1}\right)\right)$ $\dot{X}_{2}-\dot{v}=-\lambda_{2}\left(X_{2}-v\right)$

which is exponentially stable.

For simulation, we consider $D_{1}=1 \mathrm{~s}, D_{2}=2 \mathrm{~s}$ and $d=1$ and choose $\lambda_{1}=\lambda_{2}=1$. We choose a discrete sampling time equal to $10 \mathrm{~ms}$ and approximate integrals involved in predictions with a Backward Euler scheme.

Fig. 1 depicts the corresponding results. After being turned on at $t=3 \mathrm{~s}$, the control kicks in at time $t=5 \mathrm{~s}$ which is consistent with the input-delay value. This can be observed through the fact that $X_{2}$ remains to its zero equilibrium before

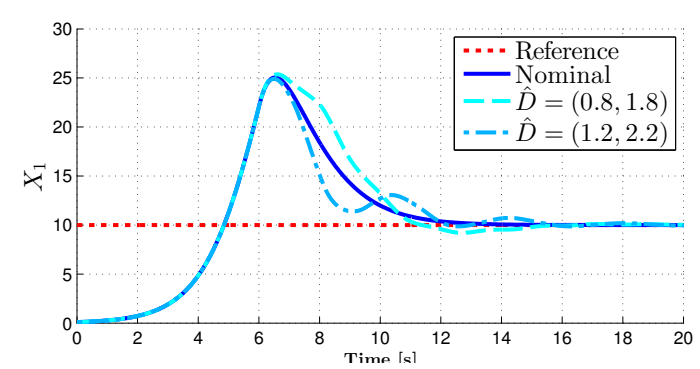

(a) Evolution of the prey population.

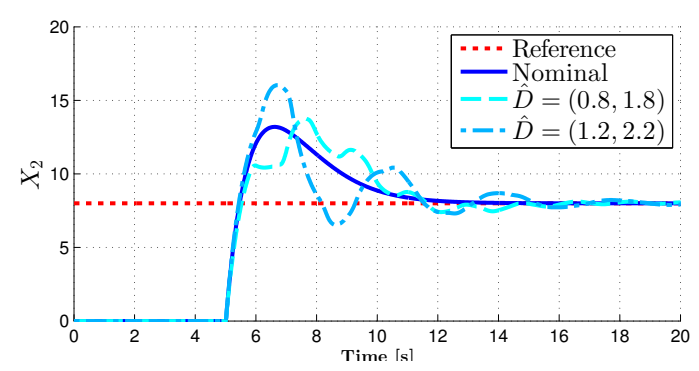

(b) Evolution of the predator population.

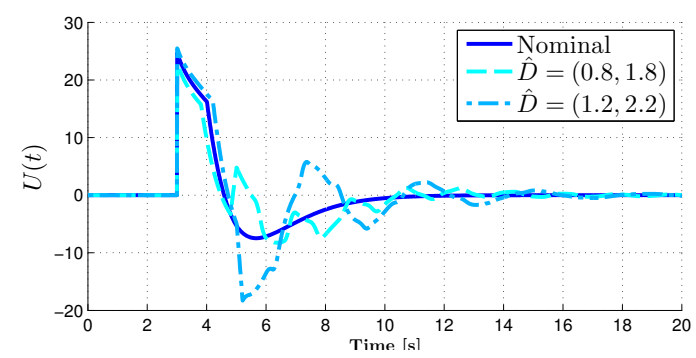

(c) Control.

Fig. 1. Simulation results for the closed-loop system consisting of the plant (64) and the control law (58),(65) with $g(x)=1-\frac{x}{50}$, $p(x)=\frac{2}{10+x}, d=1, D_{1}=1 \mathrm{~s}, D_{2}=2 \mathrm{~s}, \lambda_{1}=\lambda_{2}=1$ and the initial condition $X(0)=\left[\begin{array}{ll}0.1 & 0\end{array}\right]$. Control is turned on at $t=3 \mathrm{~s}$. We consider three cases: (i) the state- and input- delays are known; (ii) both delays are underestimated with $\hat{D}=\left(\hat{D}_{1}, \hat{D}_{2}\right)=(0.8,1.8)$; and (iii) both delays are overestimated with $\hat{D}=\left(\hat{D}_{1}, \hat{D}_{2}\right)=(1.2,2.2)$.

that time. Then, the first effect on the first coordinate can be noticed at time $t=6 \mathrm{~s}$ which is also consistent with the state delay value. Finally, one can check that asymptotic stabilization is achieved with a smooth (tunable) transient when delays are known. This transient worsens with delay uncertainties but, as Claim 1 state it, stabilization is still achieved for sufficiently small delay estimation errors.

To improve corresponding transient performances and potentially increase delay robustness, existing delay-adaptive techniques involving time-varying estimates (see [5], [6]) for both delays in the same time should be investigated within the new framework proposed in this paper in future works.

\section{ACKNOWLEDGEMENT}

The authors are indebted to Iasson Karafyllis for crucial advice to carry out the infinity-norm Lyapunov analysis.

\section{REFERENCES}

[1] Z. Artstein. Linear systems with delayed controls: a reduction. IEEE Transactions on Automatic Control, 27(4):869-879, 1982. 
[2] N. Bekiaris-Liberis. Simultaneous compensation of input and state delays for nonlinear systems. Systems and Control Letters, 73:96102, 2014

[3] N. Bekiaris-Liberis and M. Krstic. Nonlinear Control Under Nonconstant Delays, volume 25. Society for Industrial and Applied Mathematics, 2013.

[4] L. Berezansky, E. Braverman, and L. Idels. Delay differential logistic equation with harvesting. Mathematical and Computer Modelling, 40(13):1509-1525, 2004.

[5] D. Bresch-Pietri, J. Chauvin, and N. Petit. Adaptive control scheme for uncertain time-delay systems. Automatica, 48:1536-1552, 2012.

[6] D. Bresch-Pietri and M. Krstic. Delay-adaptive predictor feedback for systems with unknown long actuator delay. IEEE Transactions on Automatic Control, 55(9):2106-2112, 2010.

[7] D. Bresch-Pietri and M. Krstic. Delay-adaptive control for nonlinear systems. IEEE Transactions on Automatic Control, 59:1203-1218, 2014.

[8] D. Bresch-Pietri, T. Leroy, J. Chauvin, and N Petit. Practical delay modeling of externally recirculated burned gas fraction for sparkignited engines. In 11th Workshop on Time-Delay Systems, 2013.

[9] D. Bresch-Pietri and N. Petit. Prediction-based control for linear systems with input-and state-delay-robustness to delay mismatch. In IFAC World Congress 2014, pages 11410-11418, 2014.

[10] J. Chauvin, G. Corde, and N. Petit. Transient control of a diesel engine airpath. In American Control Conference, pages 4394-4400, 2007.

[11] T. Erneux. Applied Delay Differential Equations, volume 3. Springer Verlag, 2009.

[12] K. Gu and S. I. Niculescu. Survey on recent results in the stability and control of time-delay systems. Journal of Dynamic Systems, Measurement, and Control, 125:158, 2003.

[13] M. Jankovic. Recursive predictor design for linear systems with time delay. In American Control Conference, pages 4904-4909, 2008.

[14] T. K. Kar. Selective harvesting in a prey-predator fishery with time delay. Mathematical and Computer Modelling, 38(3):449-458, 2003.

[15] I. Karafyllis. Stabilization by means of approximate predictors for systems with delayed input. SIAM Journal on Control and Optimization, 49(3):1100-1123, 2011.

[16] I. Karafyllis and M. Krstic. Numerical schemes for nonlinear predictor feedback. Mathematics of Control, Signals, and Systems, 26(4):519546, 2014.

[17] V. Kharitonov. An extension of the prediction scheme to the case of systems with both input and state delay. Automatica, in press, 2013.

[18] V. Kolmanovskii and A. Myshkis. Introduction to the theory and applications of functional differential equations, volume 463. Springer Science \& Business Media, 1999.

[19] M. Krstic. Boundary Control of PDEs: a Course on Backstepping Designs. Society for Industrial and Applied Mathematics Philadelphia, PA, USA, 2008.

[20] M. Krstic. Lyapunov tools for predictor feedbacks for delay systems: Inverse optimality and robustness to delay mismatch. Automatica, 44(11):2930-2935, 2008.

[21] A. Manitius and A. Olbrot. Finite spectrum assignment problem for systems with delays. IEEE Transactions on Automatic Control, 24(4):541-552, 1979.

[22] C. Meyer, D. E. Seborg, and R. K. Wood. An experimental application of time delay compensation techniques to distillation column control. Industrial \& Engineering Chemistry Process Design and Development, 17(1):62-67, 1978.

[23] W. Michiels and S. I. Niculescu. Stability and Stabilization of TimeDelay Systems. Society for Industrial and Applied Mathematics, 2007.

[24] S. Mondié and W. Michiels. Finite spectrum assignment of unstable time-delay systems with a safe implementation. IEEE Transactions on Automatic Control, 48(12):2207-2212, 2003.

[25] J. D. Murray. Mathematical biology i: An introduction, vol. 17 of interdisciplinary applied mathematics, 2002.

[26] P. Pepe and I. Karafyllis. Converse lyapunov-krasovskii theorems for systems described by neutral functional differential equations in hale's form. International Journal of Control, 86(2):232-243, 2013.

[27] J.-P. Richard. Time-delay systems: an overview of some recent advances and open problems. Automatica, 39(10):1667-1694, 2003.

[28] O. J. M. Smith. A controller to overcome dead time. ISA Journal, 6(2):28-33, 1959.

[29] V. Van Assche, M. Dambrine, J. F. Lafay, and J. P. Richard. Some problems arising in the implementation of distributed-delay control laws. In Proceedings of the 38th IEEE Conference on Decision and Control, 1999., volume 5, pages 4668-4672, 1999. 